\title{
The Use of an Energy Principle to Define Atmospheric Balance
}

\author{
By M.J.P. Cullen \\ Meteorological Office, Bracknell, Berkshire, U.K. \\ J. Norbury \\ Mathematical Institute, 24-29 St. Giles, Oxford, U.K. \\ (Manuscript received 13 October 1986, in revised form 26 February 1987)
}

\begin{abstract}
An alternative approach to the definition of balance using an energy principle is presented. In middle latitudes it reduces to Lagrangian semi-geostrophic theory. Numerical calculations are shown which illustrate how such a balanced state can be found by iterative methods.
\end{abstract}

\section{Introduction}

The question of "balance" in the atmosphere is still important in the design of numerical forecasting systems, especially in improving data assimilation methods. Many centres follow an analysis step with explicit initialisation, usually normal mode initialisation e.g. Temperton and Williamson (1981). Continuous assimilation methods, e.g. Lyne et al. (1982), have to be designed to converge to some sort of Ibalanced state. In both these cases the definition of "balance" is not explicitly written down, but it is imagined that there is a subset of the solution of the model equations which varies slowly in time and is relevant to weather forecasting that in some sense defines "balance". This is often referred to as the "slow manifold", Leith (1980).

With increased use of higher resolution models and interest in forecasting actual weather elements such as cloud as well as the pressure distribution, it is necessary to be more precise about the meaning of "balance". A particular problem arises with the treatment of detailed vertical structure, important in forecasting clouds. The separation of normal modes into "slow" and "fast" modes only makes sense if the vertical scale is large enough for the gravity wave speed to be much greater than advection speeds. However a surface of discontinuity in the vertical, such as an inversion, can still be in thermal wind balance if its slope is given by the Margules relation. Furthermore the restriction to slowly varying solutions, at least in the Eulerian sense, would prevent the inclusion of moving fronts or other sharply structured systems.

An alternative approach to balance is to define a balanced state as the solution of a set of filtered equations. The close relationship between normal mode initialisation and quasi-geostrophic theory was described by Leith (1980). This approach can be made rigorous if the existence and uniqueness of solutions to the set of filtered equations can be proved. For practical usefulness, any filtered system used must have a solution, though possibly an uninformative one, in the tropics, and must include diabatic effects.

In middle latitudes the need to treat sharp vertical structures suggests the use of semi- 
geostrophic theory, Hoskins (1975). This theory is normally used together with the geostrophic coordinate transformation, Blumen (1981), to derive analytic solutions. Such solutions can only be obtained on an $f$ plane and break down in a finite time. However, Cullen and Purser (1984) showed that the Lagrangian equations can be solved in real space for indefinite periods of time, producing locally discontinuous solutions. Cullen, Chynoweth and Purser (1987) applied this method to mountain flows. Cullen et al. (1987) showed that this set of Lagrangian equations could be derived from an energy minimisation principle that made sense globally. At the equator the solutions reduce to a trivial static balance with zero horizontal pressure gradient. The solutions are unique if required to be statically, inertially and symmetrically stable. Convection is therefore represented as an instantaneous jump of a parcel between two equilibrium positions. In this sense the theory becomes a three-dimensional extension of parcel theory.

The above work suggests that the energy principle could be used to remove some of the limitations of the definition of balance discussed above since it is well-defined and allows the treatment of discontinuities. In section 2 we set out the types of motion admitted and excluded by the definition. In section 3 we set out numerical methods to derive balanced solutions by iteration. Section 4 includes results which illustrate that a discontinuous balanced state can be reached in a simple two-dimensional problem and that an overall balance can be reached on a hemisphere. The rate of convergence to this balance is analysed and shown to be strongly scale dependent, as would be expected from geostrophic adjustment theory, Blumen (1972). The results are discussed in section 5 .

\section{Definition of balance and properties}

Energy principle. For simplicity we set out the equations of motion using the $z$ coordinate defined by Hoskins (1975) and the form of Boussinesq approximation and simplification of the lower boundary condition used there. The primitive hydrostatic equa- tions are then

$$
\begin{aligned}
& \frac{D u}{D t}+\frac{\partial \phi}{\partial x}-f v=F_{u} \\
& \frac{D v}{D t}+\frac{\partial \phi}{\partial y}+f u=F_{v} \\
& \frac{D \theta}{D t}=Q \\
& \frac{\partial \phi}{\partial z}=\frac{g \theta}{\theta_{0}} \\
& \frac{D}{D t} \equiv \frac{\partial}{\partial t}+u \frac{\partial}{\partial x}+v \frac{\partial}{\partial y}+w \frac{\partial}{\partial z} \\
& \quad \equiv \frac{\partial}{\partial t}+u \cdot \nabla \\
& \frac{\partial u}{\partial x}+\frac{\partial v}{\partial y}+\frac{\partial w}{\partial z}=0 \\
& w=0 \quad \text { if } \quad z=0, H
\end{aligned}
$$

The notation is standard. $Q$ is a forcing term and $\theta_{0}$ a reference value of potential temperature. $F_{u}$ and $F_{v}$ are the $x$ and $y$ components of the frictional terms which depend nonlinearly on $u$. The appropriate energy integral was shown by Hoskins (1975) to be

$$
E=\int\left\{\frac{1}{2}\left(u^{2}+v^{2}\right)-\frac{g \theta z}{\theta_{0}}\right\} d V
$$

which is expressed as the difference between the actual energy and that of an isothermal basic state at rest.

To motivate the definition of balance consider the physical model of a rectangular container part full of water. The minimum energy state is for the water to be at rest with a horizontal surface. This surface can support "unbalanced" waves. Now suppose that the container is divided by a wall which rises above the water level. Slowly move this wall laterally so that the fluid levels are different on each side of the wall. The energy increases because of the work done in moving the wall. According to our definition a "balanced" evolution is for the water surfaces to stay flat at all times with a slow change in the levels. The real evolution would also contain transient surface waves.

Cullen et al. (1987) define a balanced solu- 
tlon of (2.1) to (2.7) in two stages based on the physical argument above. They seek to minimise the energy integral $E$ with respect to (instantaneous) parcel displacements. The pressure gradient has negligible effect on the momentum in an instantaneous displacement, but the Coriolis effect does not. Thus the horizontal momentum components will change according to the equations

$$
\begin{aligned}
& \dot{u}-f \dot{y}=0 \\
& \dot{v}+f \dot{x}=0,
\end{aligned}
$$

where the dot is a virtual time-derivative following the displacement. Potential temperature will be conserved, so that

$$
\dot{\theta}=0 \text {. }
$$

It is then proved that $E$ is minimised with respect to this class of displacements if the flow is in geostrophic and hydrostatic balance and is statically, inertially and symmetrically stable.

The time evolution is determined by requiring the fluid to adjust to minimise $E$ at each time instant. This naturally leads to the geostrophic momentum approximation in which the energy and momentum are replaced by their geostrophic values and the fluid trajectories are determined implicitly:

$$
\begin{aligned}
& \frac{D}{D t}\left(-f^{-1} \frac{\partial \phi}{\partial y}\right)+\frac{\partial \phi}{\partial x}-f v=F_{u} \\
& \frac{D}{D t}\left(f^{-1} \frac{\partial \phi}{\partial x}\right)+\frac{\partial \phi}{\partial y}+f u=F_{v} \\
& \frac{D}{D t}\left(g^{-1} \theta_{0} \frac{\partial \phi}{\partial z}\right)=Q
\end{aligned}
$$

together with (2.4) to (2.7). The energy integral which is minimised at each time instant is

$$
\int\left[\frac{1}{2}\left\{\left(f^{-1} \frac{\partial \phi}{\partial y}\right)^{2}+\left(f^{-1} \frac{\partial \phi}{\partial x}\right)^{2}\right\}-\frac{g \theta z}{\theta_{0}}\right] d V
$$

Accuracy of this approximation requires that the energy of the fluid motion given by the implicitly determined trajectory is close to the geostrophic energy. Hoskins (1975) states the condition as $V / f r \ll 1$, where $r$ is the radius of curvature of the trajectory. A similar theory applies to axisymmetric flow, as dis- cussed by Shutts and Thorpe (1978).

Existence and uniqueness of balanced state. Cullen and Purser (1984) prove that this method leads to a unique solution for a finite dimensional version of the problem on a convex physical domain (no mountains) and an $f$-plane. This solution may be discontinuous. It has not been shown whether the solution is unique in global geometry. Cullen, Chynoweth and Purser (1987) show that solutions are unique in the presence of mountains because of the physical requirement that no fluid can cross a barrier without first reaching the top. The general uniqueness result requires inertial, static and symmetric stability. In a moist flow where potential instability is released, the balanced solution will give a convecting solution where fluid is transferred instantly to its new equilibrium position. This illustrates the inability of a balanced formulation to resolve motion on a fast "adjustment" time scale.

Physical interpretation of balance definition. This definition of balance treats semi-geostrophic dynamics in mid-latitudes explicitly, but excludes gravity and inertial waves. Static, inertial and symmetric instability is removed by instantaneous adjustment. The effect of diabatic and frictional forcing is included and an ageostrophic response generated. Blocking of the flow by mountains will generate a downslope current which transfers mass across the mountain to a new position downstream; however the transfer between the ridge top and the downstream position is instantaneous. Drag is generated and energy dissipated. Mountain waves are excluded. If moist potential instability is released, mass is transferred at a rate determined by the larger scale flow (e.g. boundary layer convergence), the transfer between initial and final equilibrium positions is instantaneous and the convective available potential energy is dissipated. It is possible that under certain conditions a catastrophic instantaneous transfer of a large amount of mass could be predicted because of feedback between the convective mass transport and the ageostrophic 
response in the environment.

At the equator no horizontal pressure gradients are permitted except across mountains. The flow is determined implicitly by this requirement. Information is transmitted instantaneously around the equator. For example, the response to an isolated heat source is given by the equations

$$
\begin{aligned}
& \frac{\partial \theta}{\partial t}=\bar{Q}, \quad \frac{\partial \theta}{\partial x}=\frac{\partial \theta}{\partial y}=0 \\
& w \frac{\partial \theta}{\partial z}=Q-\bar{Q} ;
\end{aligned}
$$

where $\bar{Q}(z)$ is the average heating rate around the equator at a fixed $z$. This is the standard lowest order solution for the equatorial response to thermal forcing. The asymmetries calculated by Gill (1980) result from transient waves and are not represented.

The axisymmetric version of the theory must be used to explain hurricanes, and probably also certain mid-latitude depressions. It is thus necessary to be able to switch formulations when using this method to calculate a balanced state with real data.

This definition of balance only allows a limited description of low latitude flow, but the same comment applies to both the nonlinear balance equation and normal mode initialisation.

\section{Iterative calculation of balanced state}

Defining equations. The balance condition derived from equations (2.12) to (2.14), (2.6) and (2.7) can be written in the form:

$$
\begin{aligned}
& \frac{\partial}{\partial z}\left[f u \cdot \nabla\left(-f^{-1} \frac{\partial \phi}{\partial y}\right)+f \frac{\partial \phi}{\partial x}-f^{2} v-f F_{u}\right] \\
= & \frac{\partial}{\partial y}\left[-u \cdot \nabla \frac{\partial \phi}{\partial z}+\frac{g Q}{\theta_{0}}\right] \\
& \frac{\partial}{\partial z}\left[-f u \cdot \nabla\left(f^{-1} \frac{\partial \phi}{\partial x}\right)-f \frac{\partial \phi}{\partial y}-f^{2} u+f F_{v}\right] \\
= & \frac{\partial}{\partial x}\left[-u \cdot \nabla \frac{\partial \phi}{\partial z}+\frac{g Q}{\theta_{0}}\right] \\
& \frac{\partial u}{\partial x}+\frac{\partial v}{\partial y}+\frac{\partial w}{\partial z}=0 \\
& w=0 \quad \text { at } \quad z=0, H .
\end{aligned}
$$

These equations should be sufficient to define the total balanced wind field $(u, v, w)$; and have been proved to be in the case of an $f$ plane by Cullen and Purser (1984) provided that $\phi+1 / 2 f^{2}\left(x^{2}+y^{2}\right)$ is a convex function of $(x, y, z)$. This is equivalent to the ellipticity condition necessary in other formulations of balanced equations.

At each physical time $t$, the solution is advanced by using the value of $u$ from the previous timestep to give a provisional solution at time $t+\Delta t$. This solution will not satisfy (3.1) to (3.4). It must therefore be corrected. In this paper we use an iterative correction.

(i) Start with $\phi$ at time $t$ which must satisfy the condition that

$$
P(x, y, z)=\phi+\frac{1}{2} f^{2}\left(x^{2}+y^{2}\right)
$$

is a locally convex function everywhere, where $(x, y)$ are local horizontal Cartesian coordinates. This condition ensures that the geopotential corresponds to a physically stable solution. Applying this condition at the equator shows that $\phi$ has to take the simple form

$$
\phi=\phi_{0}(z)+y^{2} \phi_{1}(x, z)
$$

where $y$ is the local north-south coordinate.

(ii) Calculate

$$
\begin{aligned}
& v_{g}=f^{-1} \frac{\partial \phi}{\partial x}, \quad u_{g}=-f^{-1} \frac{\partial \phi}{\partial y}, \\
& \theta=\frac{\theta_{0}}{g} \frac{\partial \phi}{\partial z}
\end{aligned}
$$

The values of $u_{g}$ and $v_{g}$ are bounded at the equator because of (3.5), but only the zonal mean of $u_{g}$ has any physical meaning as a wind there. It is easily seen that $v_{g}$ is zero at the equator.

(iii) Make a first estimate $u_{g 1}, v_{g 1}, \theta_{1}$ of $u_{g}, v_{g}, \theta$ at time $t+\Delta t$ using a first guess value $u_{1}$ of $u$ which satisfies the continuity equation:

$$
\begin{aligned}
& u_{g 1}(t+\Delta t) \\
= & u_{g}(t)-\Delta t\left(u_{1} \cdot \nabla u_{g}+\frac{\partial \phi}{\partial x}-f v_{1}-F_{u_{1}}\right)
\end{aligned}
$$




$$
\begin{aligned}
& v_{g 1}(t+\Delta t) \\
= & v_{g}(t)-\Delta t\left(u_{1} \cdot \nabla v_{g}+\frac{\partial \phi}{\partial y}+f u_{1}-F_{v_{1}}\right) \\
& \theta_{1}(t+\Delta t)=\theta(t)-\Delta t\left(u_{1} \cdot \nabla \theta-Q\right) .
\end{aligned}
$$

The values of $u_{g 1}, v_{g_{1}}, \theta_{1}$ will not be consistent with the thermal wind relation, and so the value $u_{1}$ must be corrected.

(iv) Calculate a new estimate $u_{2}$ of $u$ as follows, by applying a correction depending on the residual in the thermal wind relations, scaled by a pseudo time increment $\Delta \tau$ which controls the convergence rate. The method of choice of $\Delta \tau$ is discussed below.

$$
\begin{aligned}
& \frac{\partial u_{2}}{\partial z}=\frac{\partial u_{1}}{\partial z}-\Delta \tau\left(\frac{g}{\theta_{0}} \frac{\partial \theta_{1}}{\partial x}-f \frac{\partial v_{g 1}}{\partial z}\right) \\
& \frac{\partial v_{2}}{\partial z}=\frac{\partial v_{1}}{\partial z}-\Delta \tau\left(\frac{g}{\theta_{0}} \frac{\partial \theta_{1}}{\partial y}+f \frac{\partial u_{g 1}}{\partial z}\right) .
\end{aligned}
$$

At $z=0$

$$
\begin{aligned}
& \frac{\partial}{\partial y}\left(u_{2}-u_{1}\right)-\frac{\partial}{\partial x}\left(v_{2}-v_{1}\right) \\
= & \Delta \tau\left(\frac{\partial}{\partial y}\left(f v_{g 1}\right)+\frac{\partial}{\partial x}\left(f u_{g 1}\right)\right) \\
& \frac{\partial}{\partial x}\left(u_{2}-u_{1}\right)+\frac{\partial}{\partial y}\left(v_{i}-v_{1}\right)=0 . \\
& w_{2}=-\int_{0}^{z}\left(\frac{\partial u_{2}}{\partial x}+\frac{\partial v_{2}}{\partial y}\right) d z .
\end{aligned}
$$

(v) Make new estimates $u_{g_{2}}, v_{g_{2}}, \theta_{2}$ of $u_{g}, v_{g}$ and $\theta$ at time $t+\Delta t$ :

$$
\begin{aligned}
u_{g 2}= & u_{g 1}-\Delta t\left(\left(u_{2}-u_{1}\right) \cdot \nabla u_{g}-f\left(v_{2}-v_{1}\right)\right. \\
& \left.-F_{u_{2}}+F_{u_{1}}\right) \\
v_{g_{2}}= & v_{g_{1}}-\Delta t\left(\left(u_{2}-u_{1}\right) \cdot \nabla v_{g}+f\left(u_{2}-u_{1}\right)\right. \\
& \left.-F_{v_{1}}+F_{v_{2}}\right) \\
\theta_{2}= & \theta_{1}-\Delta t\left(u_{2}-u_{1}\right) \cdot \nabla \theta .
\end{aligned}
$$

Repeat to convergence to specified tolerance. The value of $\phi(t+\Delta t)$ at $z=0$ is determined from the values of $u_{g}$ and $v_{g}$ there.

Properties of the iteration. The properties can be illustrated most easily by considering a two-dimensional problem. Suppose that at time $t$ the solution is $\phi=\phi^{0}(x, z)$ with $\theta^{0}$ and $v_{g}^{0}$ determined from (3.6). The effect of solving equations (3.7) to (3.9) can be considered as adding an unbalanced perturbation to this basic state. The convergence of the iteration will then mirror the evolution of this perturbation according to the linearised primitive equations, since the equations (3.10) and (3.11) for $\left(u_{2}-u_{1}\right)$ and $\left(v_{2}-v_{1}\right)$ take the same form as equations (2.1) and (2.2) for $D u / D t$ and $D v / D t$. Assuming a solution proportional to $e^{i \sigma \tau}$, the analysis of Hoskins (1974) gives

$$
\sigma^{2}=N^{2} \sin ^{2} A-2 s^{2} \sin A \cos A+f \zeta \cos ^{2} A
$$

where

$$
\begin{aligned}
& N^{2}=\frac{g}{\theta_{0}} \frac{\partial \theta^{0}}{\partial z}, \quad s^{2}=\frac{g}{\theta_{0}} \frac{\partial \theta^{0}}{\partial x}, \\
& \zeta=f+\frac{\partial v_{g}^{0}}{\partial x}
\end{aligned}
$$

and $A$ is the disturbance orientation measured from the horizontal. Therefore, if the potential vorticity

$$
q=f \zeta N^{2}-s^{4}
$$

is negative, the perturbations amplify and the iteration will diverge. It is therefore necessary to ensure that the data presented to the iteration is statically, symmetrically and inertially stable. Ideally a slantwise convective adjustment (Emanuel, 1983) should be performed first.

The convergence is achieved by wave dispersion and the rate will depend on the frequency $\sigma$. The approach to the solution will be determined in the same way as in standard geostrophic adjustment (Blumen, 1972). Because the range of possible frequencies is very large, the rate of convergence will be highly scale dependent. The generation of waves during the iteration may lead to initial divergence from the solution on scales associated with low frequencies. In the explicit iteration set out in (3.10) to (3.16), the scaling factor $\Delta \tau$ is restricted by the highest frequency in the same way as the timestep is restricted in a primitive equation model.

Practical implementation. The above presentation has been in $z$ coordinates to simplify the equations. This does not allow prediction 
of the surface pressure. For practical implementations the scheme has been used in $\sigma$ coordinates, with iteration also required to satisfy surface geostrophic balance and the constraint (3.12) removed. The iteration as set out above is explicit, it can also be reformulated using a standard semi-implicit algorithm to allow the "time step" $\Delta \tau$ to be increased. In this case the values $\theta_{1}, u_{g 1}$, and $v_{g 1}$ in equations (3.10) and (3.11) and (3.12) are replaced by values $\theta_{2}, u_{g_{2}}, v_{g_{2}}$ defined by equations (3.14) to (3.16). The resulting implicit system of equations is then linearised and decomposed in the standard way, e.g. Hoskins and Simmons (1975). This method is analogous to taking a backward implicit time step when solving the primitive equations, which leads to damping of gravity waves. In our case it leads to damping of the residuals in equations (3.10) and (3.11) and hence faster convergence.

\section{Numerical results}

Two-dimensional case. We first illustrate illustrate the difference between an explicit and backward semi-implicit implementation of the iteration. The data used are for a

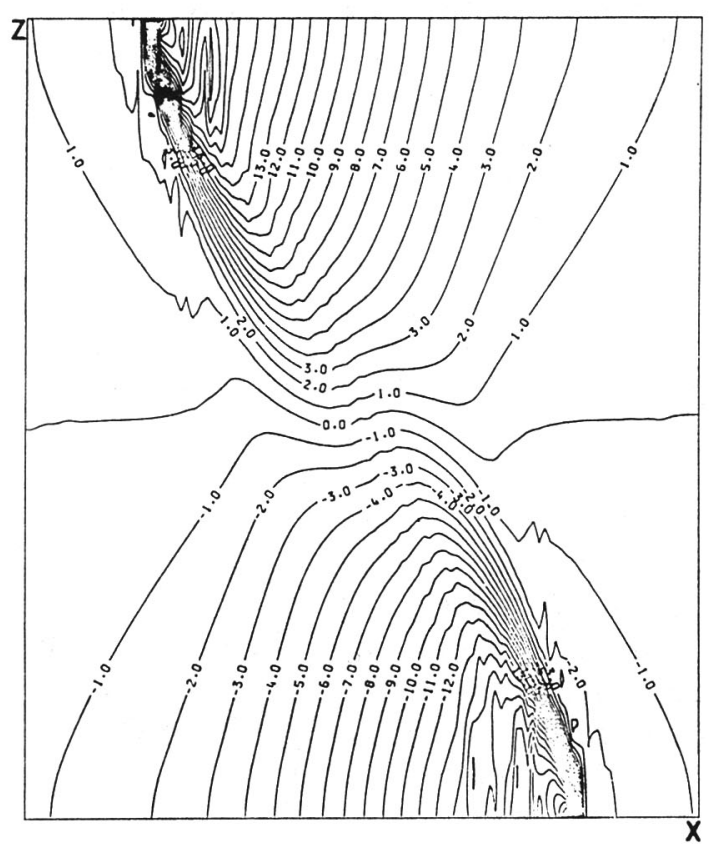

Fig. 1 The $y$-component of geostrophic wind obtained by integrating two-dimensional frontal model. Units $\mathrm{ms}^{-1}$. two-dimensional frontal model as described in Cullen and Purser (1984), with discontinuities forming at the lower and upper boundaries and peak geostrophic winds of $20 \mathrm{~ms}^{-1}$ as shown in Fig. 1. The residual after explicit iteration is shown in Fig. 2 as a plot of

$$
v_{g}-f^{-1} \frac{\partial \phi}{\partial x}
$$

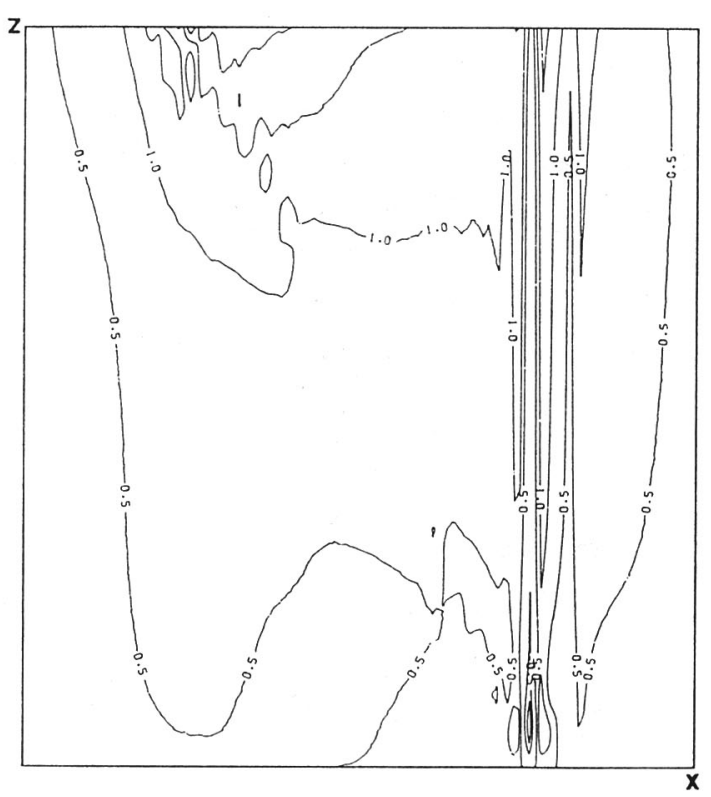

Fig. 2 Error in iteration to thermal wind balance in two-dimensional model using explicit iteration. Units $\mathrm{ms}^{-1}$

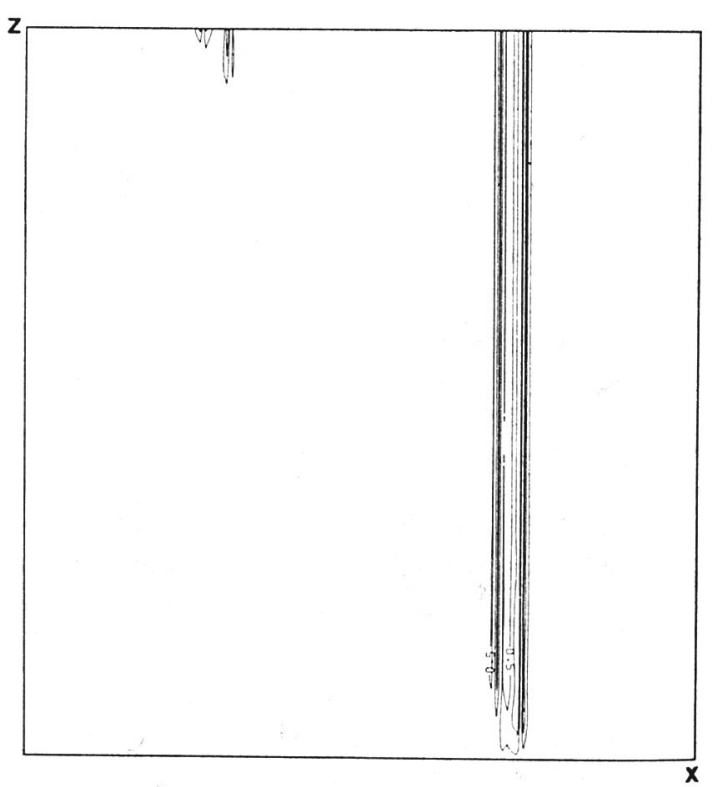

Fig. 3 As Fig. 2 using semi-implicit iteration. 
with $v_{g}$ given by equation (3.15) and $\phi$ by vertical integration of (3.16). There are large values at the surface and upper front of the residual in the thermal wind and a large value of the residual in the surface geostrophic balance. Away from these areas is a large region where there is a residual of $1 \mathrm{~ms}^{-1}$, about 5 per cent of the maximum $v_{g}$. Using the same number of implicit iterations, Fig. 3 , removes almost all the residual except near the front. Doubling the number of explicit iterations gave similar results to Fig. 3 . Thus a useful improvement in convergence rate has been achieved.

Three-dimensional case. We illustrate the residuals for a hemispheric implementation. The initial residuals are generated by one 15 minute time step of the form (3.7) to (3.9) including diabatic effects. The implicit version of the iteration was used and the convergence analysed by splitting the residual
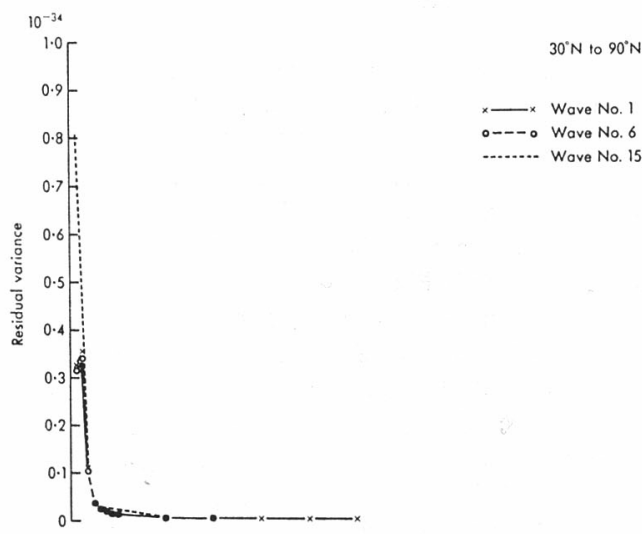
----o Wave No. 6 -........ Wave No. 15

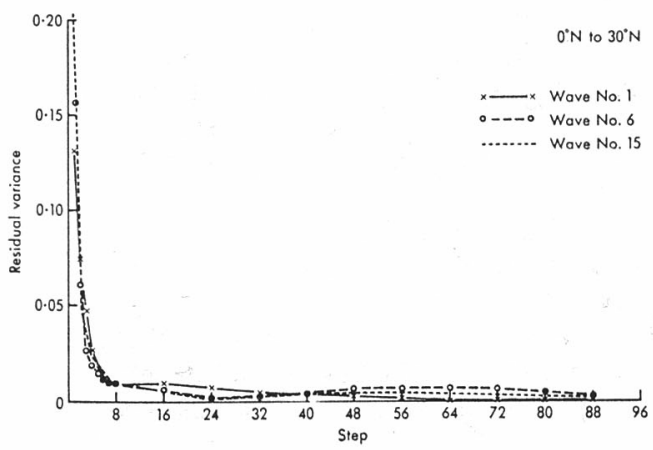

Fig. 4 Residual variance in iteration to balanced data projected onto external mode. Three zonal wavenumbers are shown: (a) area averaged from $30^{\circ} \mathrm{N}$ to $90^{\circ} \mathrm{N}$, (b) from $0^{\circ} \mathrm{N}$ to $30^{\circ} \mathrm{N}$.

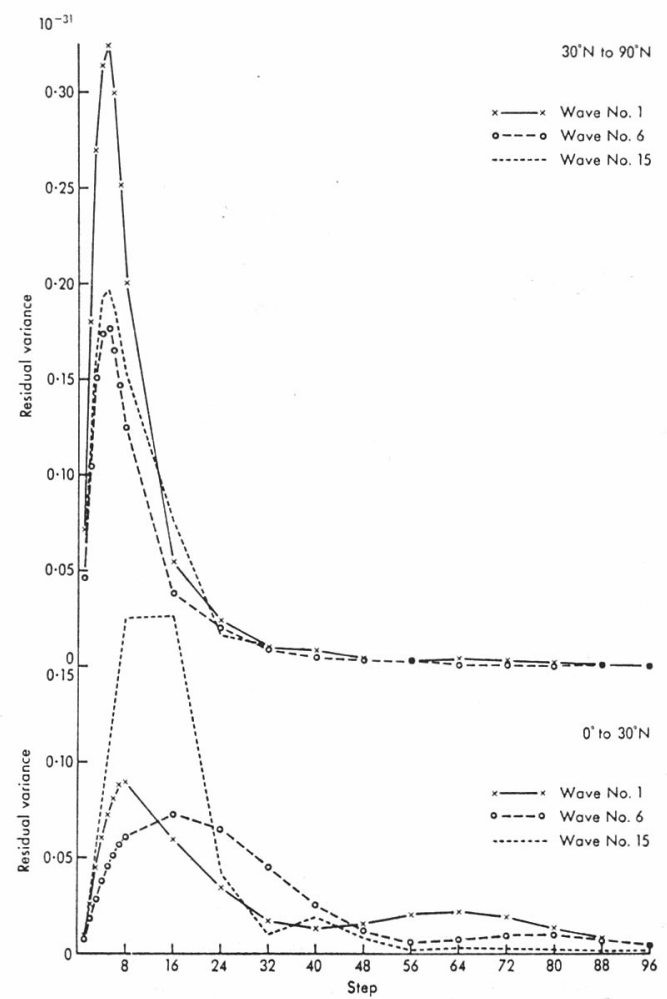

Fig. 5 As Fig. 4 but for fourth internal mode.

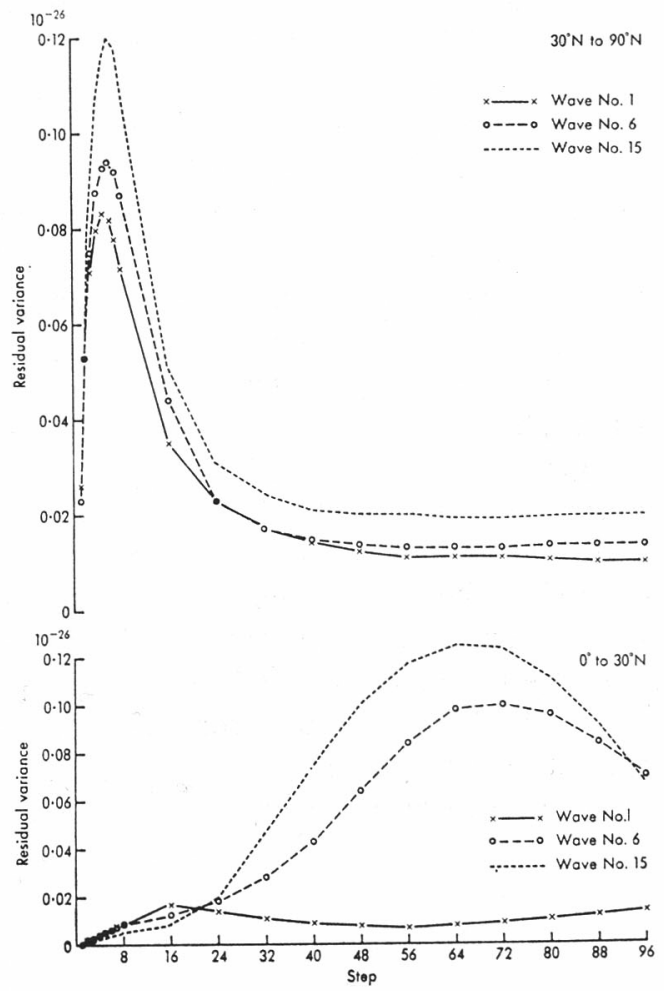

Fig. 6 As Fig. 4 but for highest internal mode. 
into the vertical normal modes appropriate to an isothermal basic state of $300^{\circ} \mathrm{K}$. The variance in each mode is plotted against number of iterations for zonal wave numbers 1,6 and 15 averaged over the regions $0^{\circ}$ to $30^{\circ} \mathrm{N}$ and $30^{\circ}$ to $90^{\circ} \mathrm{N}$. Convergence in the external mode, Fig. 4, occurs within a few iterations, irrespective of horizontal scale and latitude. Convergence in the fourth internal mode, Fig. 5, takes of the order of 8 "hours" and the residuals increase during the first 4 "hours" of iteration. In low latitudes convergence takes over 12 "hours". In the highest internal mode, Fig. 6, convergence takes 12 "hours" in middle latitudes but never really takes place in low latitudes. This behaviour is entirely consistent with linear geostrophic adjustment theory.

\section{Discussion}

The fact that convergence could be obtained in the hemispheric iteration apart possibly from the highest resolvable mode, which is mostly noise, is consistent with the existence of the balanced state discussed in section 2. However the convergence rate is very slow and it is likely that direct rather than iterative methods will be needed to compute it in practice. A possible approach is discussed by Cullen et al. (1987). In an integration of a primitive equation model the solution will attempt to maintain a balanced state by generating an ageostrophic circulation in a very similar way to the iteration used above. Thus if the time scale of the dynamical and physical forcing is shorter than the convergence time scale of the iteration, a balanced response is not to be expected and a free unbalanced wave response will be generated. The diagnostic study of Errico (1984) illustrates this for the NCAR model. The scales on which balance is maintained agree with those expected from the convergence rate shown in this paper.

It is an open question whether this scale dependence of the ability of the atmosphere to maintain balance is a correct physical description of the atmosphere or is a defect of current model formulations. It is hoped that further work will clarify this important issue.

\section{References}

Blumen, W., 1972: Geostrophic adjustment. Rev. Geoph. Space Phys., 10, 485-528.

1981: The geostrophic coordinate transformation. J. Atmos. Sci., 38, 1100-1105.

Cullen, M. J.P. and R. J. Purser, 1984: An extended Lagrangian model of semi-geostrophic frontogenesis. J. Atmos. Sci., 41, 1477-1497.

, S. Chynoweth and R. J. Purser, 1987 : On semi-geostrophic flow over synoptic scale topography. Quart. J. Roy. Meteor. Soc., 113, 163180.

, J. Norbury, R. J. Ptrser and G. J. Shutts, 1987: Modelling the quasi-equilibrium dynamics of the atmosphere. To appear in Quart. J. Roy. Meteor. Soc., 113 (July issue).

Errico, R.M., 1984: The dynamical balance of a general circulation model. Mon. Wea. Rev., 112, 2439-2454.

Gill, A.E., 1980: Some simple solutions for heatinduced tropical circulation. Quart. J. Roy. Meteor. Soc., 106, 447-462.

Hoskins, B. J., 1974: The role of potential vorticity in symmetric stability and instability. Quart. J. Roy. Meteor. Soc., 100, 480-482.

- 1975: The geostrophic momentum approximation and the semi-geostrophic equations. J. Atmos. Sci., 32, 233-242.

- and A.J. Simmons, 1975: A multi-layer spectral model and the semi-implicit method. Quart. J. Roy. Meteor. Soc., 101, 637-655.

Leith, C. E., 1980: Nonlinear normal mode initialisation and quasi-geostrophic theory. J. Atmos. Sci., 37, 958-968.

Lyne, W.H., R. Swinbank and N.T. Birch, 1982 : A data assimilation experiment and the global circulation during the FGGE special observing periods. Quart. J. Roy. Meteor. Soc., 108, 575594.

Shutts, G. J. and A. J. Thorpe, 1978: Some aspects of vortices in rotating stratified fluids. Pageoph., 116, 993-1006.

Temperton, C. and D. L. Williamson, 1981: Normal mode initialisation for a multilevel grid-point model. Mon. Wea. Rev. 109, 729-743. 